\title{
Medical Image of the Week: DBS Polysomnogram Artifact
}

\author{
Safal Shetty, MD \\ Kenneth S. Knox, MD
}

Pulmonary, Allergy, Critical Care \& Sleep Medicine

Banner University Medical Center

Tucson, AZ

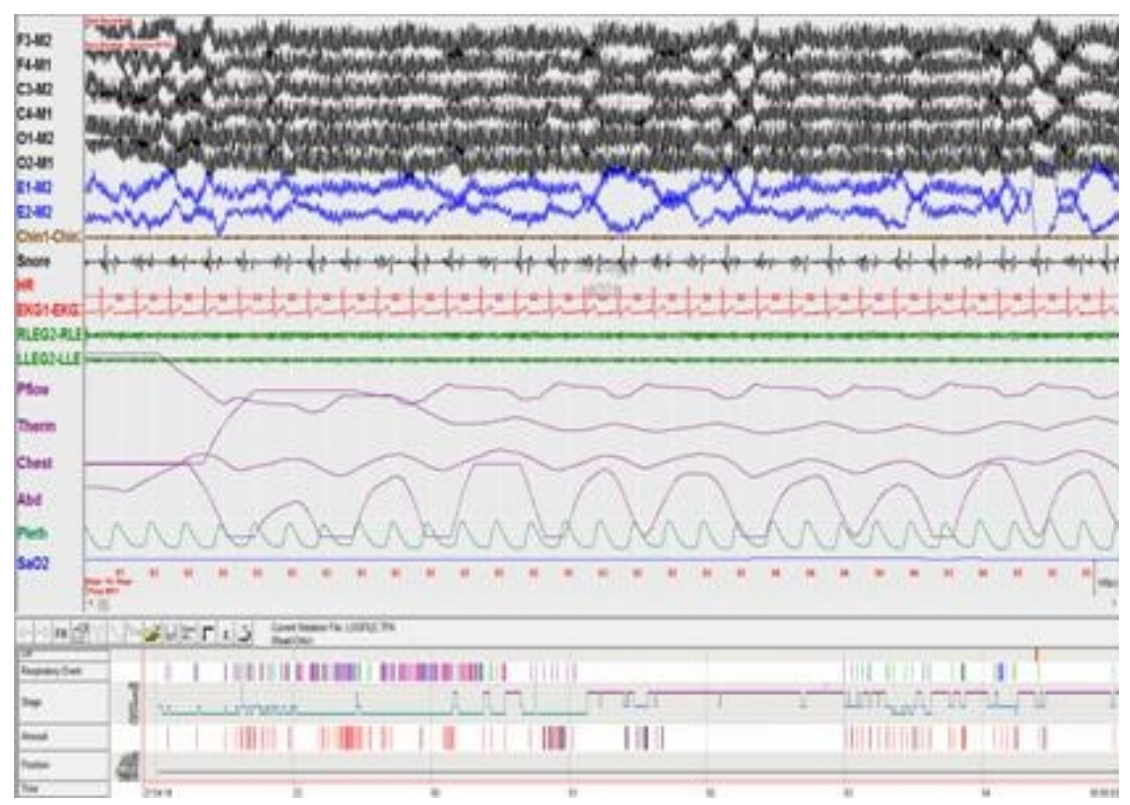

Figure 1. Thirty second epoch showing DBS artifact obscuring all recording channels except flow, efforts belts and the EKG channels.

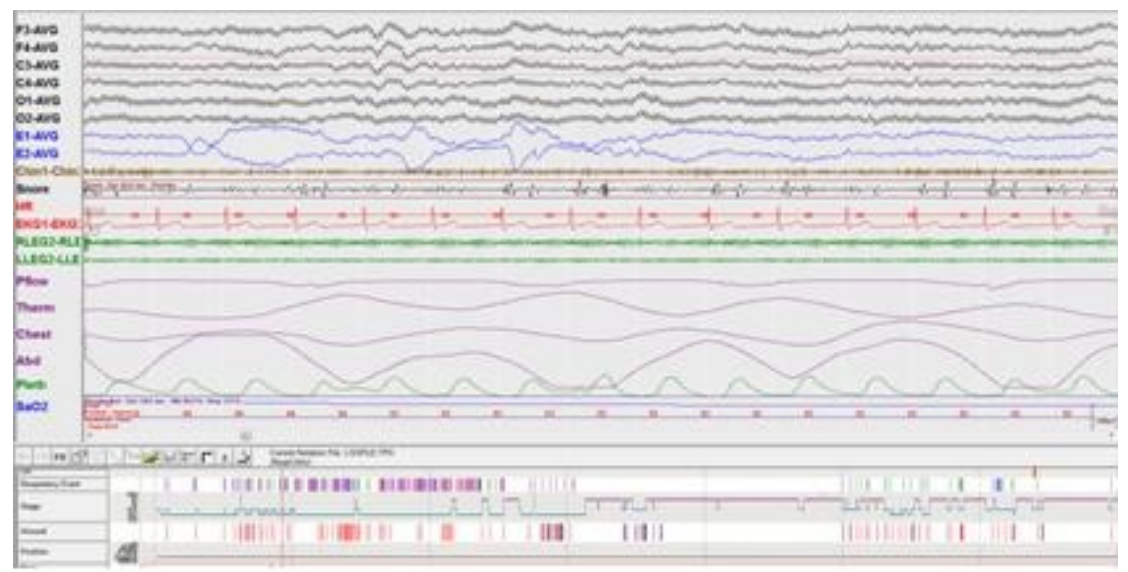

Figure 2. Ten second epoch showing the electrical artifact due to DBS. 
A 79-year-old man with known Parkinson's disease and status post deep brain stimulator (DBS) implantation underwent an overnight polysomnogram for clinical suspicion of obstructive sleep apnea. Artifact was seen on the polysomnogram recording (Figures 1 \& 2).

Patient-related electrical artifacts may be seen from devices such as pacemakers, deep brain stimulators and vagal nerve simulators. Abrupt discontinuation of DBS is associated with a high likelihood of worsening of symptoms in patients with Parkinson's disease (1). Patients with DBS are most commonly programmed in monopolar mode. Bipolar configuration, forms a short electrical dipole that affects a relatively smaller volume of tissue and generates far less artifact, suggesting that this may be an effective option in a Parkinsonian patient with indications for polysomnography (2).

\section{References}

1. Chou KL, Siderowf AD, Jaggi JL, Liang GS, Baltuch GH. Unilateral battery depletion in Parkinson's disease patients treated with bilateral subthalamic nucleus deep brain stimulation may require urgent surgical replacement. Stereotact Funct Neurosurg. 2004;82(4):153-5. [CrossRef] [PubMed]

2. Frysinger RC, Quigg M, Elias WJ. Bipolar deep brain stimulation permits routine EKG, EEG, and polysomnography. Neurology. 2006;66(2):268-70. [CrossRef] [PubMed] 